\title{
The Low-Temperature Ring during Droplet Impact on a Superhydrophilic Surface
}

\author{
Huixia Ma ${ }^{1}$, Jiang Chun ${ }^{2}{ }^{\infty}$, Feng Zhou ${ }^{1}$, Kai Qiao ${ }^{1}$, Rui Jiang ${ }^{1}$, Shumei Zhang ${ }^{1}$ and Tingting Hao ${ }^{2, *}$ \\ 1 Dalian Research Institute of Petroleum and Petrochemicals, SINOPEC, Dalian 116045, China; \\ mahuixia.fshy@sinopec.com (H.M.); zhoufeng.fshy@sinopec.com (F.Z.); qiaokai.fshy@sinopec.com (K.Q.); \\ jiangrui2018.fshy@sinopec.com (R.J.); zhangshumei.fshy@sinopec.com (S.Z.) \\ 2 Liaoning Key Laboratory of Clean Utilization of Chemical Resources, Dalian University of Technology, \\ Dalian 116024, China; chunjiang_dlut@126.com \\ * Correspondence: haotingting224@dlut.edu.cn
}

check for updates

Citation: Ma, H.; Chun, J.; Zhou, F.; Qiao, K.; Jiang, R.; Zhang, S.; Hao, T. The Low-Temperature Ring during Droplet Impact on a Superhydrophilic Surface. Coatings 2021, 11, 1043. https://doi.org/10.3390/coatings 11091043

Academic Editor: Rahmat Ellahi

Received: 13 July 2021

Accepted: 27 August 2021

Published: 30 August 2021

Publisher's Note: MDPI stays neutral with regard to jurisdictional claims in published maps and institutional affiliations.

Copyright: (c) 2021 by the authors. Licensee MDPI, Basel, Switzerland. This article is an open access article distributed under the terms and conditions of the Creative Commons Attribution (CC BY) license (https:// creativecommons.org/licenses/by/ $4.0 /)$
Abstract: Droplet impact on the solid surfaces is widespread in nature, daily life, and industrial applications. The spreading characteristics and temperature evolution in the inertial spreading regime are critical for the heat and mass transfer process on the solid-liquid interface. This work investigated the spreading characteristics and temperature distribution of the thin liquid film in the inertial rapid spreading regime of droplet impact on the heated superhydrophilic surfaces. Driven by the inertial and capillary force, the droplet rapidly spreads on the superhydrophilic surface, resulting in a high temperature center in the impact center surrounded by a the low-temperature ring. The formation of the unique the low-temperature ring on the heated superhydrophilic surface is due to the much smaller time scale of rapid spreading than that of heat transfer from the hot solid surface to the liquid film surface. CFD numerical simulation shows that the impacting droplet spreads and congests in the front of liquid film, leading to the formation of vortex velocity distribution in the liquid film. Increasing We number and wall temperature can accelerate the heat transfer rate of liquid film and shorten the existence time of the low-temperature ring. The findings of the the low-temperature ring on the superhydrophilic surface provide the guidelines to optimization of surface structures and functional coatings for enhancing heat transfer in various energy systems.

Keywords: superhydrophilic surface; droplet impact; liquid film spreading; low-temperature ring

\section{Introduction}

Droplet impact on the solid surface is the typical problem of free-surface flow. Due to the high efficiency of phase change heat transfer, the droplet impact process is widely used in various industrial applications, e.g., electronic cooling [1], desalination [2], chemical engineering, nuclear industry, and refrigeration. The interaction of impacting droplet and solid surface play a significant role in the heat transfer efficiency of spray cooling. A water droplet that is deposited on a superhydrophilic surface can spread into a thin liquid film quickly, which is preferred for enhancing heat transfer efficiency. Superwettability and capillary wicking of the superhydrophilic surface make it a major candidate for spray cooling technology. Since the phenomenon of droplet impacting was experimentally studied by Worthington in 1876 [3], the subject continues to attract research scholars of various disciplines [4]. The emphasis has been on spreading, bouncing, splashing, bubble entrapment, and droplet atomization after droplet impact on the solid surfaces [4-6]. The impact of the droplets on the solid surfaces is affected by inertial force, surface tension, viscous force, roughness, and wettability of the solid surfaces [7,8] while the impact dynamics can directly affect the heat transfer efficiency between the wall and the droplet. In addition, wall temperature can influence the spreading and retraction behaviors of the droplets on solid surfaces [9-12]. Alizadeh et al. [13] found that the retraction phenomenon after droplet impact on the hydrophilic surface is highly dependent on the temperature. 
The lower the wall temperature, the higher the viscosity; the higher viscous dissipation and surface adhesion during the retraction process, the slower the retraction process would occur. Jin et al. $[14,15]$ studied the behavior of droplets impinging on the cold solid surface. The results show that different from the impact on hot temperature surface, the droplets would not retract after spreading to the maximum spreading diameter while the droplets would freeze faster on the supercooled surface with the increase in impact We number. Most of the earlier work focusing on droplet spreading and infiltration behaviors on the thin nanostructured coatings and porous surfaces $[16,17]$, such as the superhydrophilic micropillar arrays [18], textured superhydrophilic surfaces [19], natural porous stone [17], and nanotube surfaces $[20,21]$. Taking advantage of numerical simulation, e.g., Lattice Boltzmann method [22-24], level set method [25], and molecular dynamic simulation [26,27], many researchers have investigated the droplet spreading and penetration process on the porous surface. Chen et al. [28] investigated the spreading and capillary wicking effect that is occurred on the nanostructured surface and further compared the relationship between the time and the spreading as well as capillary wicking radius. Due to the irregular nanochannel size distribution, the nanostructure surface with irregular nanowires shows faster liquid spreading and wicking rates than that on the surface with regular nanowires. Wemp et al. [29] established a model to predict the spreading rate of the wetted footprint of a deposited water droplet and defined transition conditions between synchronous and hemispreading regimes. The results indicate that the early synchronous spreading regime is characterized by the flow primarily localized near the upper droplet contact line in the porous layer. Wang et al. [30] investigated the water droplet spreading and imbibition on a superhydrophilic poly (butylene terephthalate) melt-blown fiber affected by hydrolysis time and surface topologies. In addition, they established the mathematical model and compared it with measured permeabilities obtained through numerical simulation. Epsin et al. [31] proposed a model based on the lubrication theory of droplet spreading on permeable substrates. This model incorporates surface roughness to describe the contact-line pinning that is observed in experiments.

Many studies of droplet impact on the solid surface have focused on the hydraulic characteristics of the liquid spreading as reviewed. However, there is a lack of understanding of the temperature evolution after a droplet impacting a hot surface. During the temperature distribution when the droplet impacts onto the heated solid surface, the temperature increases from the center to the edge, and the droplet surface temperature increases linearly with time. Girard et al. [32] investigated the temperature distribution and evolution of millimeter-scale droplets impacting the heating surface using infrared thermal imaging technology. The results show that the temperature of the droplet surface from the center to the edge gradually increases. Gao et al. [33] studied the temperature evolution and transient heat transfer process of the droplets impinging the horizontally flowing liquid film. The results show that the temperature of the liquid film surface increases from the center to the edge.

Although numerous studies have been dedicated to the droplet impact process on the solid surface, the heat and mass transfer processes are still unclear. To the best of our knowledge, the spreading characteristics and temperature distribution evolution for the droplet impact on the heated superhydrophilic surface remains unexplored, even though the process exists in many natural and industrial applications. More importantly, the temperature distribution after inertial spreading is important for the subsequent heat transfer process for the overall heat transfer process. Li et al. [34,35] found that the temperature gradient reversal phenomenon of the droplets impinging on the prewetted superhydrophilic surface. However, the interaction mechanism and underlying mechanism during droplet impact on the preexisting liquid film is qualitatively different from that on the dry superhydrophilic surface. The temperature distribution of the droplet spreading on the dry superhydrophilic surface offers a potential enhanced heat transfer process of thin liquid film evaporation, which can decrease the local hot spots and increase the heat transfer efficiency. 
In this work, the superhydrophilic surface is prepared by the chemical oxidation etching process. The spreading characteristics and temperature evolution of a cold droplet spreading on the heated dry superhydrophilic surface are experimentally investigated by a high-speed camera and infrared thermal imager simultaneously. The flow behaviors of the liquid film in the inertial spreading regime on the superhydrophilic surface are also investigated to illustrate the heat and mass transfer mechanism. The influences of the initial wall temperature and Weber number on the temperature distribution evolution are discussed. It is found that the low-temperature ring is formed during the droplet impact on the superhydrophilic surface, which is due to the cold liquid film congestion when liquid film spreads on the superhydrophilic surface. Furthermore, CFD numerical simulation is employed to reveal the mechanism of the low-temperature ring formation.

\section{Experimental Method}

Copper is widely used in thermal systems because of its high thermal conductivity. Superhydrophilic surface is defined as the surface whose water contact angle is lower than $10^{\circ}$. The copper-based superhydrophilic surface is commonly fabricated using the oxidative etching method. The copper material and oxidative etching method are used in this work. The superhydrophilic surface is prepared by a chemical oxidative etching method on the copper surface [36,37]. The fabrication procedure is briefly described as follows. The top surface of the copper block is carefully polished with emery paper (from \#400 to \#3000), rinsed with deionized water, then ultrasonically washed with acetone, ethanol, deionized water, and dried with nitrogen. Then, it is etched in a preheated alkaline solution composed of $\mathrm{NaClO}_{2}, \mathrm{NaOH}, \mathrm{Na}_{3} \mathrm{PO}_{4} \cdot 12 \mathrm{H}_{2} \mathrm{O}$, and deionized water (3.75:5:10:100 wt $\left.\%\right)$ at $96 \pm 2{ }^{\circ} \mathrm{C}$ for 15 min. Scanning Electron Microscope (NOVA Nano SEM 450, FEI, Hillsboro, OR, USA) images of the hydrophilic surface and superhydrophilic surface are shown in Figure 1. An SEM image of $\mathrm{CuO}$ nanostructures shows that the fabricated $\mathrm{CuO}$ film has unique knifelike morphologies with a height of $1 \mu \mathrm{m}$. The contact angle is measured by a contact angle meter (OCA25, Data physics, Filderstadt, Germany) at room temperature using the sessile drop method. As shown in Figure 2, the contact angle after the chemical oxidative etching is $2.9 \pm 2^{\circ}$ while the smooth hydrophilic copper surface is about $76.6 \pm 5^{\circ}$.

(a)

(b)
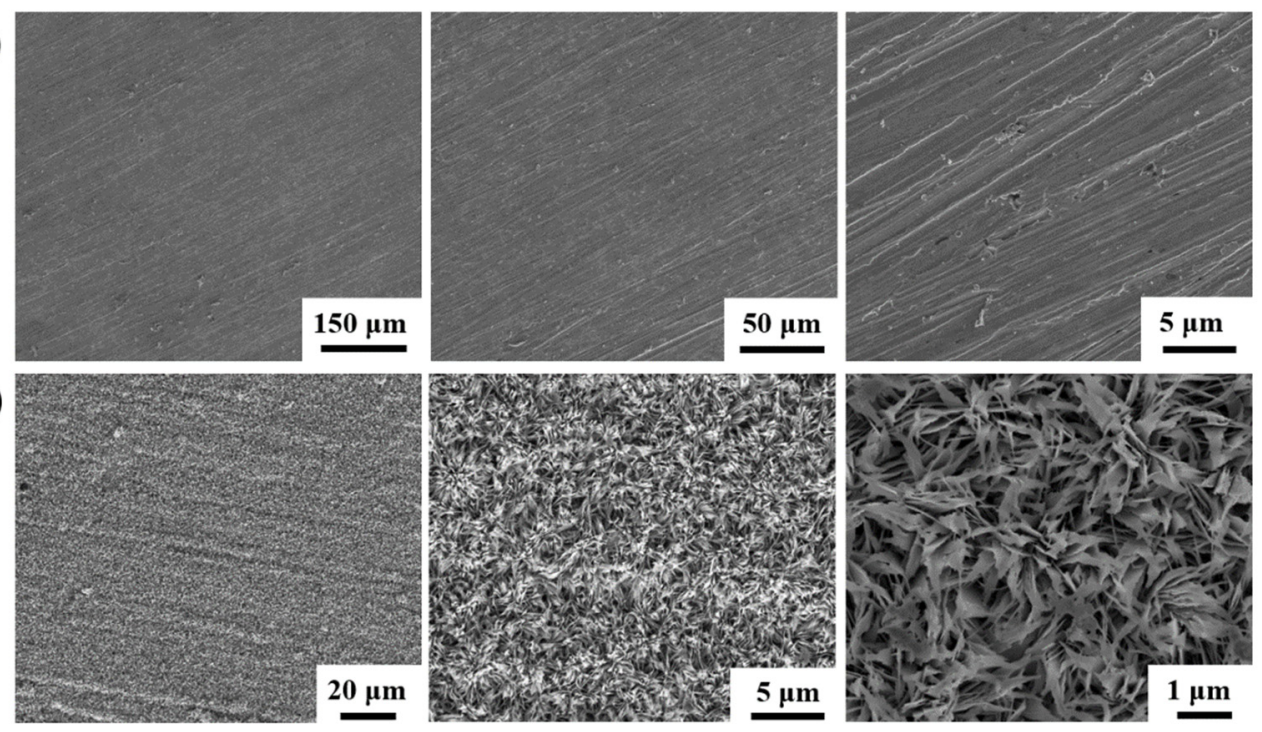

Figure 1. SEM images of (a) smooth hydrophilic surface (b) micro/nanostructured superhydrophilic surface. 


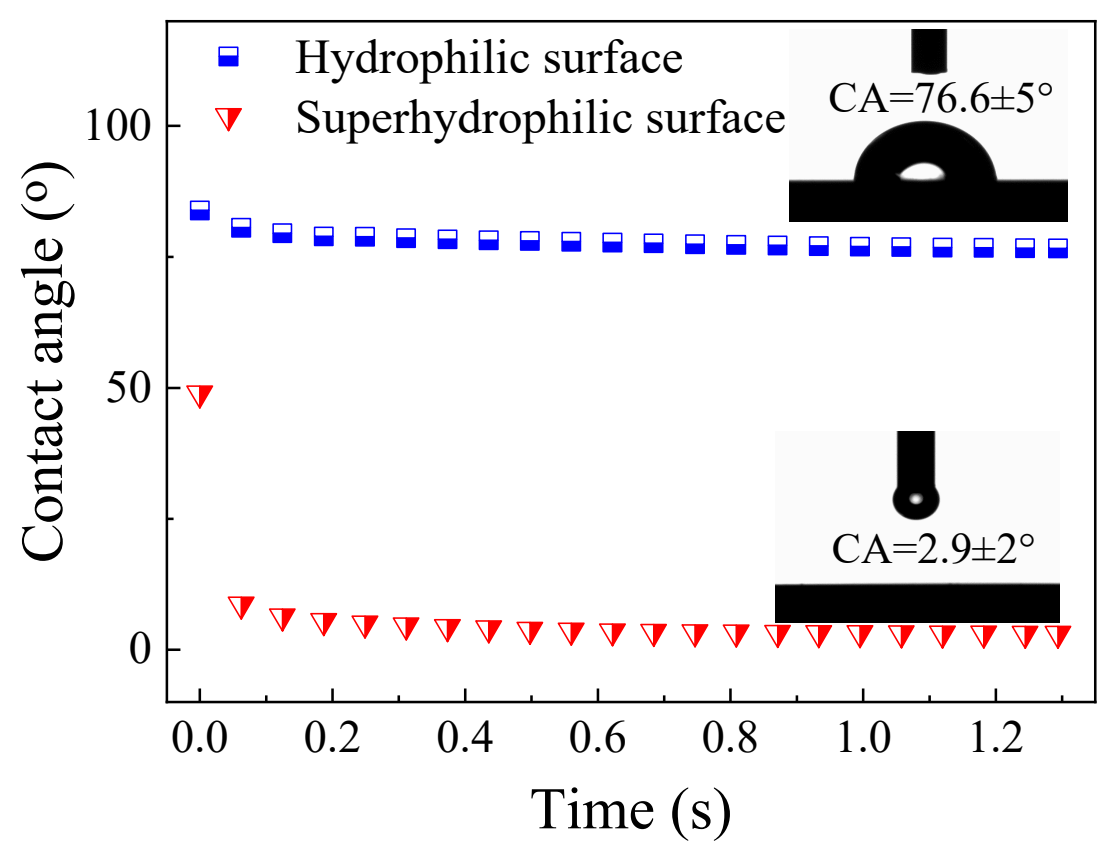

Figure 2. Contact angle measurement of hydrophilic and superhydrophilic surfaces.

The experimental setup of the droplet impact system is shown in Figure 3. It consists of five following parts: (1) two high-speed cameras at the same frame rate of $5000 \mathrm{fps}$; (2) a heating system; (3) droplet generation device including a microsyringe pump and $30 \mathrm{G}$ needle; (4) an infrared thermal imager with the frame rate of $50 \mathrm{fps}$; (5) a temperature measurement system; (6) a light source. The droplet impact platform consists of a constant temperature platform and a collision platform as shown in Figure 3. A high-speed camera was kept at the platform level, shooting from the positive front view, and set the other high-speed camera was set obliquely shooting the droplet impact process from the oblique upper direction. The liquid film temperature distribution was obtained by an infrared thermal imager. In the process of ascertaining the emissivity value of water, we changed the emissivity constantly until the temperature measured by the infrared thermal imager matched the thermocouple reading in real-time. Duplicate experiments are conducted to obtain the emissivity value of water, which was about 0.96 . Deionized water was pumped into the droplet injector through a syringe pump, and the flow rate of the syringe pump is controlled to change the droplet dropping frequency for experimental measurement. The impact platform was a cylindrical copper block with a diameter of $30 \mathrm{~mm}$ and a height of $11 \mathrm{~mm}$, and four holes of $1 \mathrm{~mm}$ diameter with equal spacing for temperature measurement. The upper surface of the copper block was exposed to the air, and the bottom surface was in contact with the heating stage and coated with thermally conductive silicone oil. The heat preservation device is a hollow block of tetrafluoroethylene (PTFE). To prevent the accumulation of liquid film on the heat preservation device, the impact copper block is higher than $1.5 \mathrm{~mm}$. The linear relationship of four thermocouple values proved that the heat preservation device has a better heat insulation effect. The wall temperature $T_{\mathrm{w}}$ was obtained by thermocouple with the uncertainty of $\pm 0.05{ }^{\circ} \mathrm{C}$ at the near-wall surface due to the higher thermal conductivity of copper. All thermocouples were calibrated in a constant temperature bath (FLUKE9171, Fluke, Everett, WA, USA). The automatic linear slide controled the distance between the needle and the target surface to change the impact velocity of the droplet. The droplet impact velocities have a standard deviation of about $\pm 0.5 \mathrm{~mm} / \mathrm{s}$. The droplet impact velocity was expressed by Weber number in the following discussion. The We number was the ratio of kinetic energy to surface energy. The We number and droplet equivalent diameter are expressed as:

$$
W e=\frac{\rho v^{2} d_{\text {drop }}}{\sigma}
$$




$$
d_{\mathrm{drop}}=\left(d_{\mathrm{h}}^{2} d_{\mathrm{v}}\right)^{\frac{1}{3}}
$$

where $d_{\mathrm{h}}, d_{\mathrm{v}}$ represent the horizontal and vertical diameters of the droplets respectively. $d_{\text {drop }}$ is the drop diameter, $v$ is the impact velocity of the droplet. Water liquid density $\rho=998 \mathrm{Kg} / \mathrm{m}^{3}$, and water-air surface tension $\sigma=0.0728 \mathrm{~N} / \mathrm{m}^{-1}$. The values of $d_{\mathrm{h}}, d_{\mathrm{v}}$ are measured from the high-speed image by Image-Pro Plus. The pixel measurement error is a pixel size of $0.0248 \mathrm{~mm}$. The measured droplet size is $2.23 \mathrm{~mm}$, and the relative error is $1.11 \%$. The experimental conditions are normal pressure, the droplets are all deionized water, the surrounding and droplet temperature is $20^{\circ} \mathrm{C}\left( \pm 2{ }^{\circ} \mathrm{C}\right)$, and relative humidity is about $50 \%( \pm 5 \%)$. Because of the short time of spreading and wicking, the evaporation of the droplet was neglected.

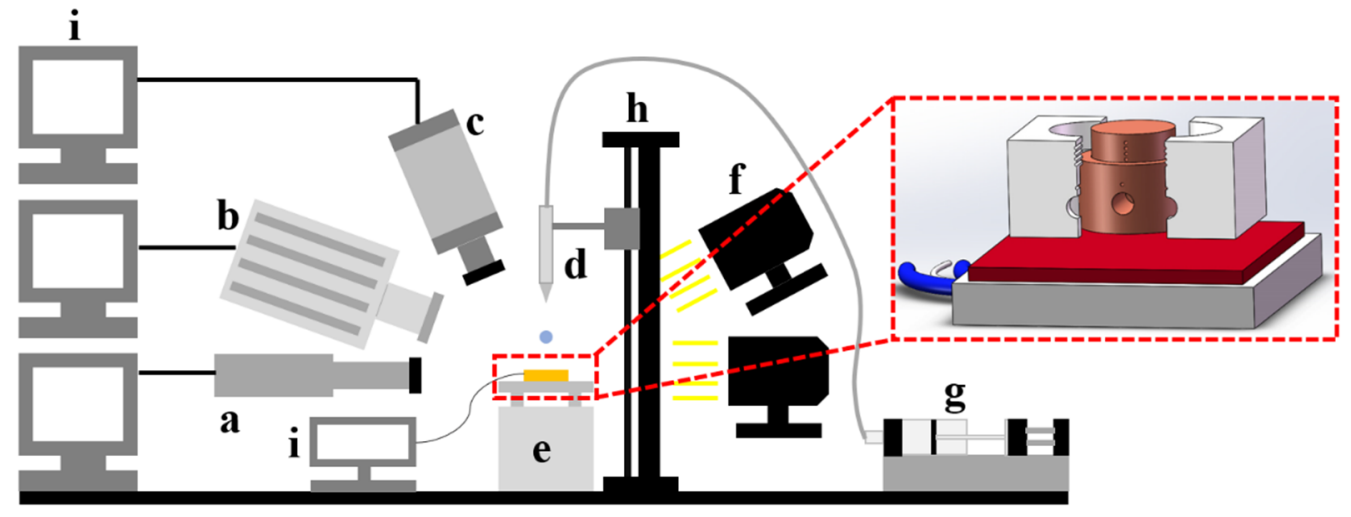

Figure 3. Experimental system of droplet impact. Two high speed cameras (a,b); (c) infrared thermal imager; (d) droplet generating device; (e) heating platform; (f) light source; (g) microsyringe pump; (h) linear displacement slide; (i) data acquisition computer.

\section{Results}

\subsection{The Low-Temperature Ring on the Heated Superhydrophilic Surface}

The inertial spreading and oscillating behavior of the droplet on the hydrophilic copper surface are displayed in Figure 4 . The droplets are in a regular spherical shape before the droplet impacts the copper surface, as shown in $t=0 \mathrm{~ms}$ in Figure 4 . Once contacting the surface, the droplet will spread driven by inertia force to the maximum spreading diameter. Then, the droplet oscillates under the action of surface tension and viscous force until reaching an equilibrium state eventually after a period of oscillation process, as shown in $t=10-60 \mathrm{~ms}$ in Figure 4. The temperature distribution after the inertial spreading regime is shown in Figure 5. The appearance of the cold droplet for temperature distribution is regarded as the initial time of the temperature distribution evolution results. It can be seen that the temperature gradually increases from the impact center to the border during the spreading process. This kind of temperature distribution is caused by the droplet shape distribution during the spreading and oscillating process of the droplet. When a droplet spreads on a solid surface, the heat transfer process of the droplet spreading on solid surfaces is mainly dominated by the heat convection in the inertial spreading regime, while the subsequent heat transfer process is mainly dominated by heat conduction. As compared with the heat transfer timescale, the spreading timescale is short and can be ignored during the droplet impact spreading process. Therefore, the heat conduction after spreading mainly occupies the main proportion of the overall heat transfer of the liquid film. Then for the heat conduction process of static liquid film, the initial temperature distribution and the thickness distribution of liquid film determine the heat transfer time. The low-temperature is located at the region with thicker liquid film thickness on the hydrophilic surface, and this kind of temperature distribution and liquid film thickness distribution is the most unfavorable. The heat transfer process of the liquid film can be shortened if the region with the lowest temperature is transferred from the thickest region to the relatively thin region of the liquid film. 


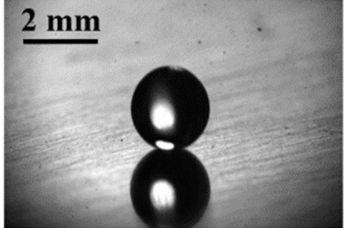

$0 \mathrm{~ms}$

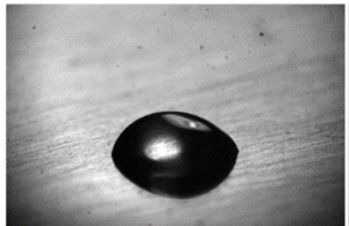

$30 \mathrm{~ms}$

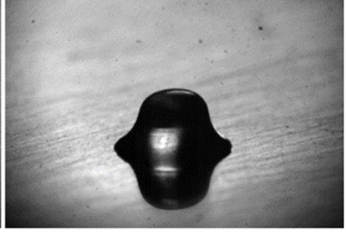

$10 \mathrm{~ms}$

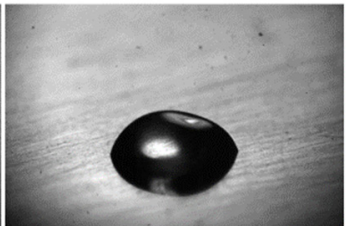

$40 \mathrm{~ms}$

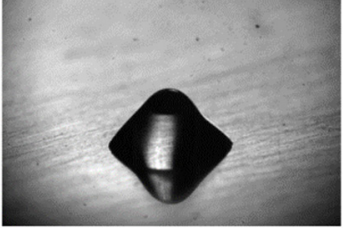

$20 \mathrm{~ms}$

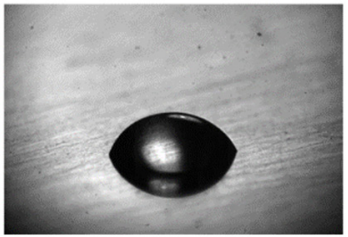

$60 \mathrm{~ms}$

Figure 4. Droplet impact and spreading on the hydrophilic copper surface in different stages. Experimental conditions: $\mathrm{We}=7.6, \mathrm{~T}_{\mathrm{W}}=40^{\circ} \mathrm{C}$.
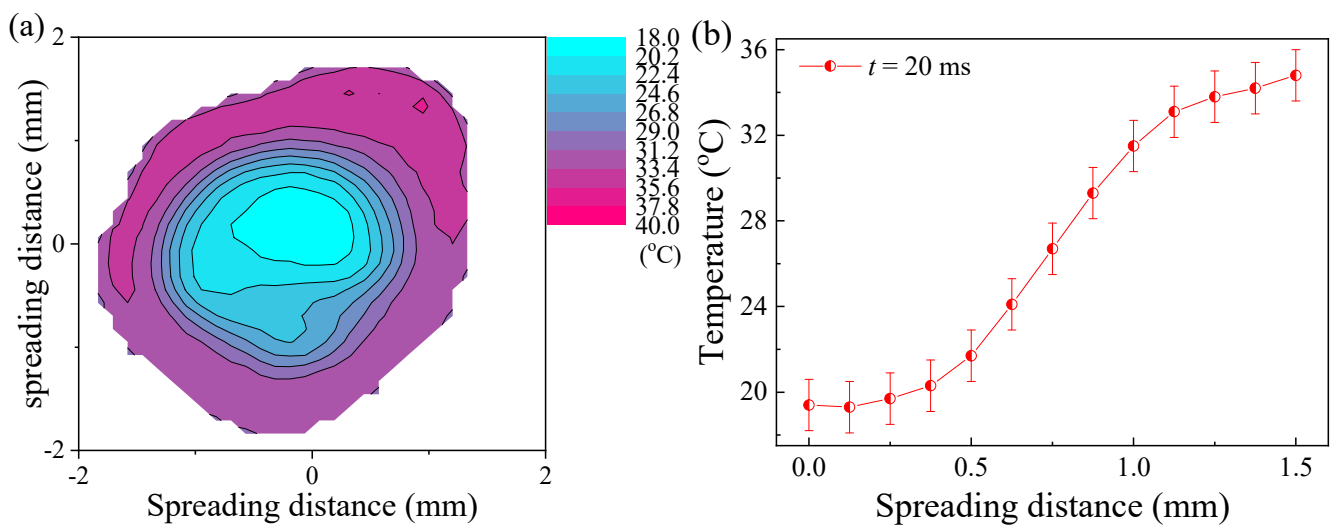

Figure 5. Temperature distribution of droplet impact on the hydrophilic copper surface at $t=20 \mathrm{~ms}$. (a) Infrared image and (b) cold and hot temperature regions. Experimental conditions: $W e=7.6, T_{\mathrm{w}}=40^{\circ} \mathrm{C} .0$ represents the impact center.

As shown in Figure 6, in the initial stage $(t=10 \mathrm{~ms})$ that the droplet contacts with the superhydrophilic surface, the lower part of the droplet rapidly spreads driven by the inertial force, and the upper part of the droplet remains spherical under the surface tension. The upper part of the droplet gradually forms a cone from the spherical depression, and then the conical water column on the liquid film shrinks and disappears. At $t=20 \mathrm{~ms}$, the liquid film is concave, and the center is an ultra-thin liquid film. After the inertial force disappears, the liquid film retracts driven by surface tension, and finally the central pit disappears $(t=20-40 \mathrm{~ms})$. In general, the droplet impact on the superhydrophilic surface spreads rapidly laterally. The shape of the liquid film is similar to the "crater", after which the liquid is returned to the impact center driven by the surface tension. The appearance of the change in the temperature distribution after droplet impact on the superhydrophilic surfaces is regarded as the initial time of the infrared camera result. The temperature distribution in Figure 7 clearly shows the ring-shaped the low-temperature zone at different times. The temperature distribution on the superhydrophilic surface differs from that on the hydrophilic surface with the same We number and wall temperature or previous studies of temperature distribution [32,33]. There is a significant difference between the hydrophilic surface with the same condition and temperature distribution evolution that the coldest area does not appear in the impact center. The temperature distribution shows a the low-temperature ring along the spreading radial direction. Moreover, the low-temperature rings will not disappear immediately, but will gradually stabilize with time. The average temperature of the liquid film gradually increases with time, but the special temperature trend of the low-temperature ring structure along the spreading radial 
direction does not change, decreasing first and then rising along the radial direction. The ring-shaped the low-temperature zone persists during the entire heating process. As the liquid film gradually heats up, the temperature of the entire area is almost heated to the wall temperature, and the low-temperature ring will eventually disappear. As illustrated before, the temperature distribution after the inertial spreading regime is important for the subsequent heat conduction process, which is determined by the thickness of the liquid film as the main thermal resistance. As compared with the hydrophilic surface with the same We number and wall temperature, the low-temperature region transfers from the thickest region to the relatively thin region of the liquid film. The appearance of the low-temperature ring shortens the heat transfer time for subsequent heat conduction. Therefore, the formation of the low-temperature ring on superhydrophilic surfaces is of great significance to the enhancement of heat transfer because of the lowest temperature of the liquid film surface from the thickest region to the relative thin-film region.

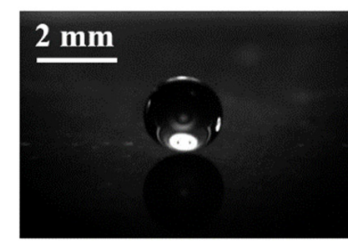

$0 \mathrm{~ms}$

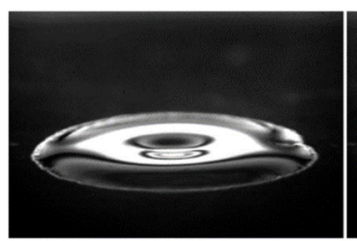

$30 \mathrm{~ms}$

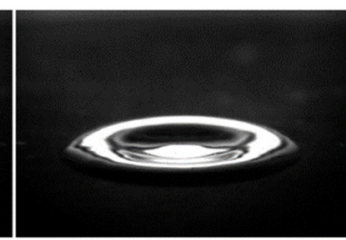

$10 \mathrm{~ms}$

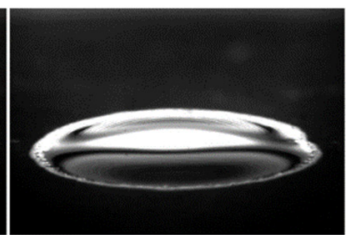

$40 \mathrm{~ms}$

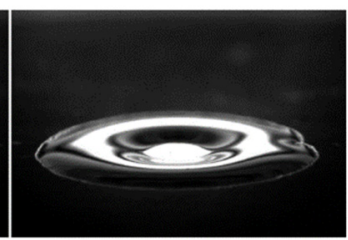

$20 \mathrm{~ms}$

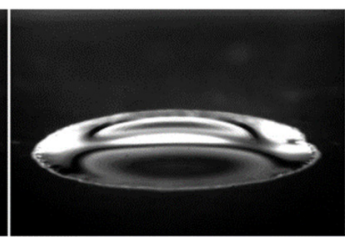

$60 \mathrm{~ms}$

Figure 6. Droplet impact and spreading on the superhydrophilic surface in different stages. Experimental conditions: $W e=7.6, T_{\mathrm{w}}=40.0{ }^{\circ} \mathrm{C}$.
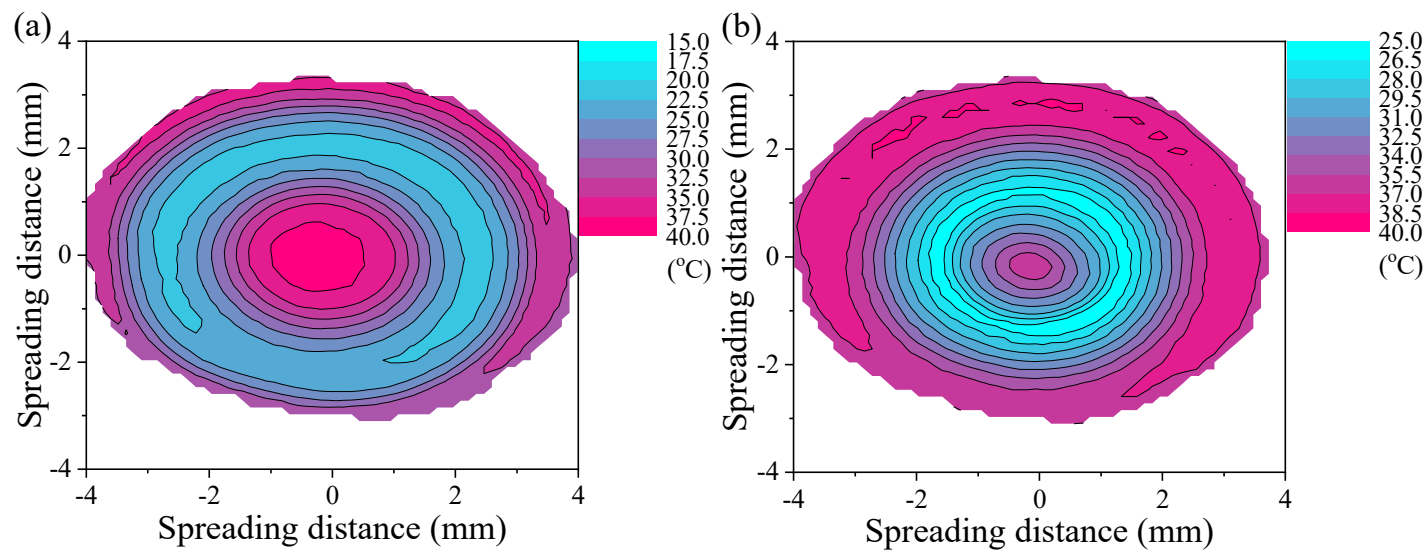

Figure 7. Cont. 

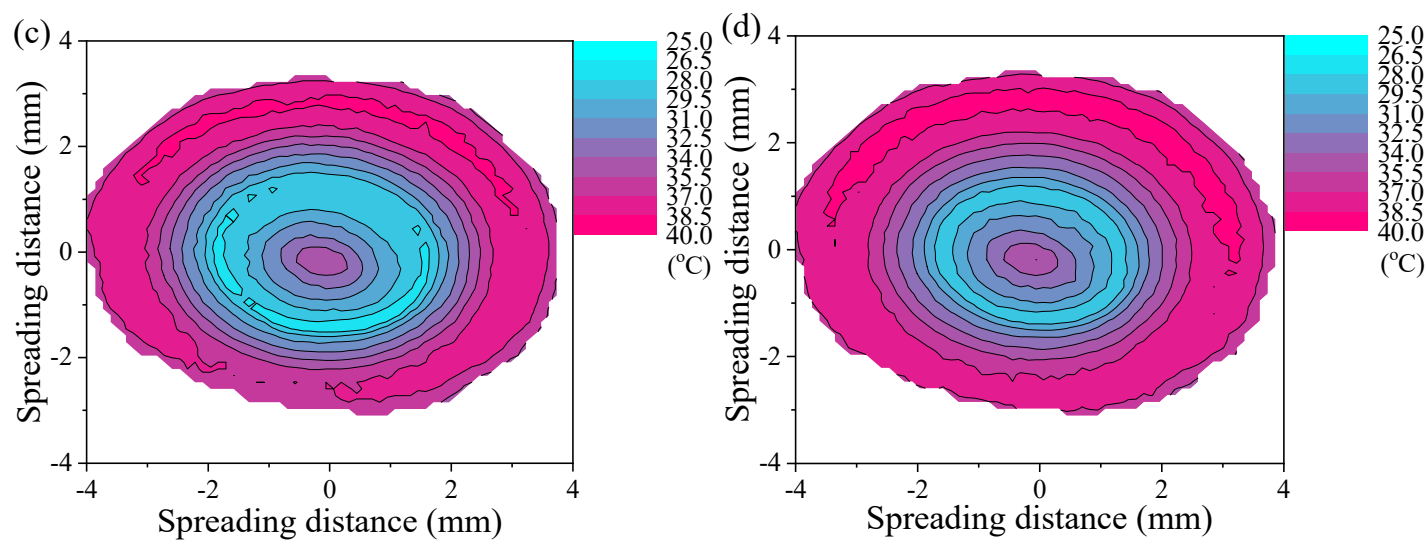

Figure 7. Temperature distribution and evolution of droplet impact on the superhydrophilic surface at (a) $t=20 \mathrm{~ms}$, (b) $t=40 \mathrm{~ms},(\mathbf{c}) t=60 \mathrm{~ms}$, and (d) $t=80 \mathrm{~ms}$. Experimental conditions: $W \mathrm{e}=7.6, T_{\mathrm{w}}=40.0^{\circ} \mathrm{C} .0$ represents the impact center.

\subsection{Temperature Evolution of the Impacting Droplet on the Heated Superhydrophilic Surface}

\subsubsection{Effect of Weber Number}

The radial temperature distributions from the impact center to edge region are shown in Figure 8 for various We numbers and times. The temperatures at the initial stage between droplet impact on the hydrophilic surface and superhydrophilic surface are similar, but the next time of temperature distribution has changed. It can be seen from Figure 8a that, the temperature at the impact center is the same as the temperature of the cold droplet at the initial time. However, different from the liquid film temperature distribution at the initial time, the radial temperature distribution is in a valley at $20 \mathrm{~ms}$, and the coldest area in the temperature distribution has changed from the center region to the ring-shaped region. The position of the coldest area moves from the center to the middle region. A valley appears in the radial temperature distribution called ring-shaped the low-temperature zone. As the heat transfer process continues, the liquid film temperature increases to wall temperature gradually, but the tendency of temperature distribution remains unchanged. The ring-shaped temperature distribution lasts throughout the entire heat transfer process. The low-temperature ring will move toward the center, then its position stays unchanged with time until the heat transfer end. The center temperature is always 4 to $15^{\circ} \mathrm{C}$ higher than the temperature of the ring-shaped cold zone for wall temperature is $40.0^{\circ} \mathrm{C}$. With the increase in impact We number, the initial position will shift outwards because the spreading diameter increases but the tendency of temperature distribution remains unchanged at different We numbers range from 7.6 to 341 . As shown in Figure 8, with the increase in impact We number, the existence time of the low-temperature ring will be shortened. The spreading time in the inertial spreading regime is ultrafast which maintaining about $20 \mathrm{~ms}$. Compared to the spreading rate of liquid film, the heat transfer rate of the liquid film is too small to be ignored. Therefore, the formation of a ring-shaped cold region of the liquid film shows the effect of cold liquid film accumulation to a great extent. 

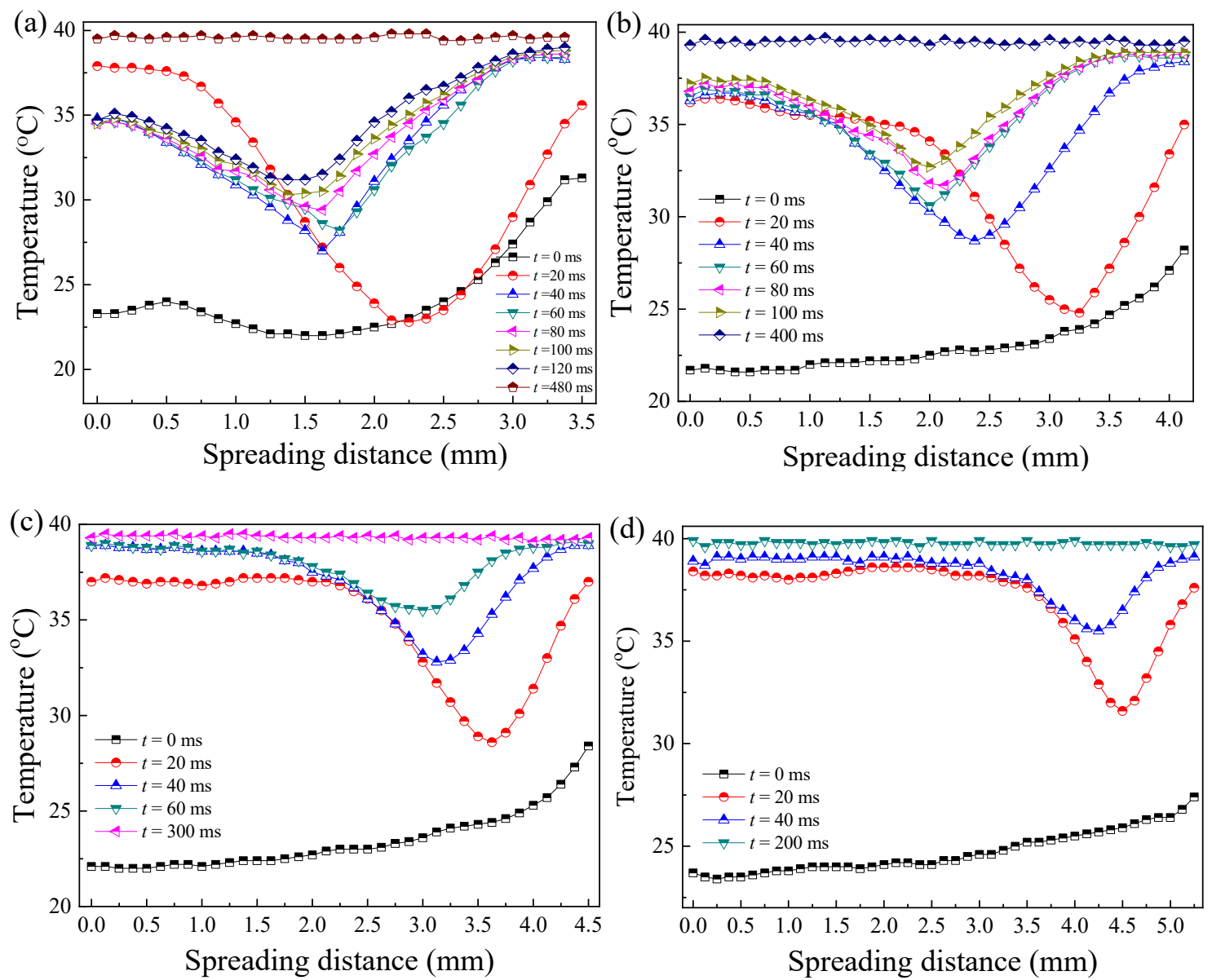

Figure 8. Radial temperature distributions of the spreading droplet on superhydrophilic surface for (a) $W e=7.6, T_{\mathrm{w}}=40.0^{\circ} \mathrm{C}$, (b) $W e=84.0, T_{\mathrm{w}}=40.0^{\circ} \mathrm{C}$, (c) $\mathrm{We}=156.5, T_{\mathrm{w}}=40.0{ }^{\circ} \mathrm{C}$, (d) $\mathrm{We}=341.3, T_{\mathrm{w}}=40.0^{\circ} \mathrm{C}$.

\subsubsection{Effect of Wall Temperature}

The radial temperature distributions from the center to edge region are shown in Figure 9 with various wall temperatures. The temperature difference between the ringshaped the low-temperature zone and the surrounding increases with the increase in the wall temperature, and the low-temperature ring exists when the wall temperature ranges from 30 to $72.5^{\circ} \mathrm{C}$. Comparing the temperature distribution curves at different times, the temperature of the low-temperature ring will gradually extend inward, and then keep its position unchanged. The initial formation position does not change with the increase of wall temperature. The existence time of the low-temperature ring decreases with the increase of wall temperature. The higher the wall temperature is, the higher the temperature gradient appears that accelerates the heat transfer rate and the shorter period in which temperature uniformity is achieved. The cold liquid film accumulated in the congested area and formed a the low-temperature ring at the thick liquid film zone which also has a large thermal resistance. Additionally, there is an ultra-thin liquid film at high temperature center. As shown in Figure 9, the temperature of the low-temperature ring gradually increases with time, and the temperature at the center of the ring rises slowly until the low-temperature ring zone disappears. 

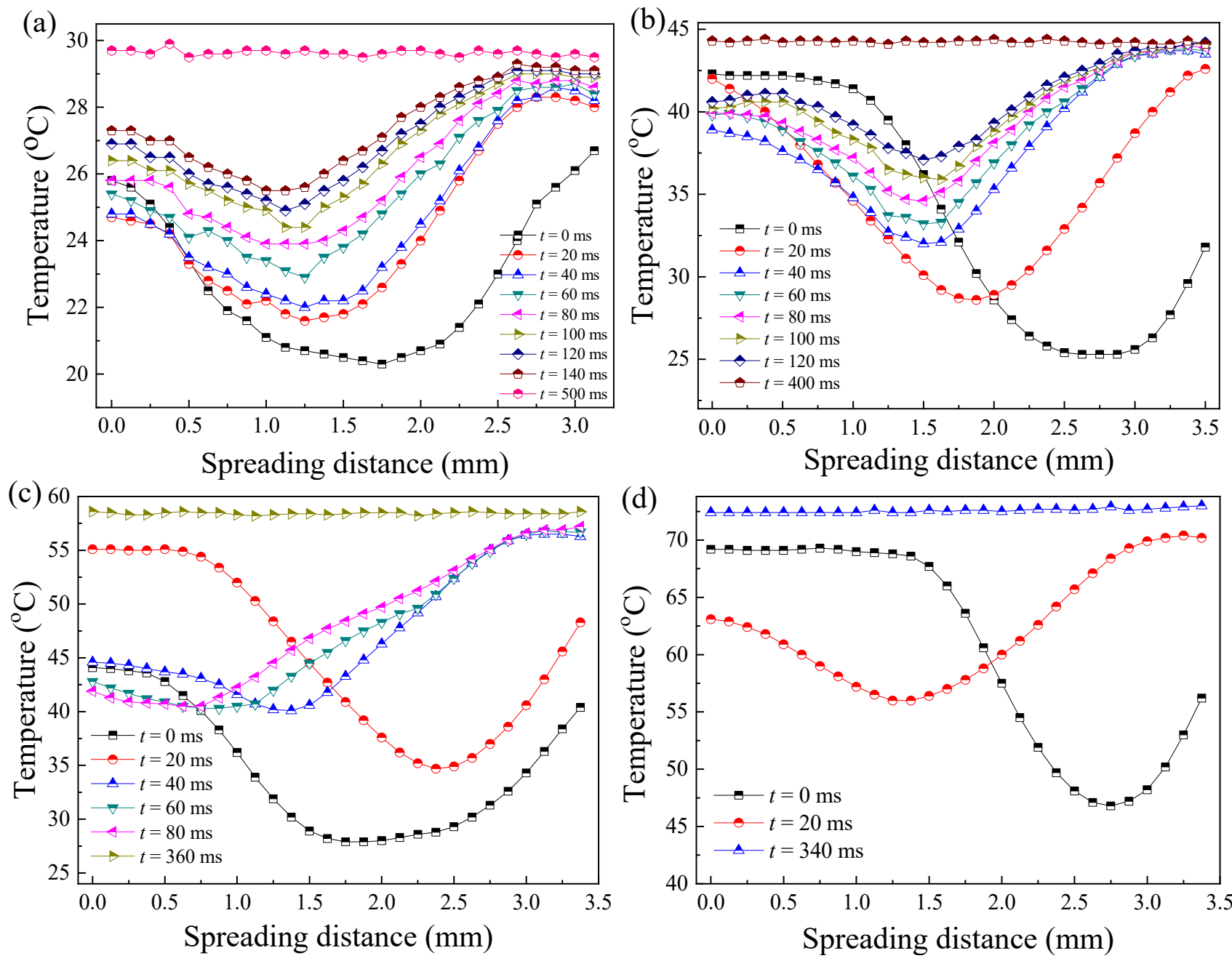

Figure 9. Radial temperature distributions of the spreading droplet on superhydrophilic surface for (a) $W e=7.6, T_{\mathrm{w}}=30.0^{\circ} \mathrm{C}$, (b) $W e=7.6, T_{\mathrm{w}}=44.0^{\circ} \mathrm{C}$, (c) $\mathrm{We}=7.6, T_{\mathrm{w}}=58.0{ }^{\circ} \mathrm{C}$, (d) $\mathrm{We}=7.6, T_{\mathrm{w}}=72.5^{\circ} \mathrm{C}$.

\subsection{Mechanism of The Low-Temperature Ring on the Superhydrophilic Surface}

CFD numerical simulations using the VOF model couple with the Level-set method were employed to investigate the mechanism for the formation of the low-temperature ring on a superhydrophilic surface. The computational domain is a two-dimensional rectangular box with dimensions of $2 \times 10 \mathrm{~mm}$, as shown in Figure S1. The model adopts the following assumptions. (1) The flow is two-dimensional, incompressible, and laminar. (2) A phase change of the droplets is not considered. Based on these assumptions, the governing equations are listed in the Supplementary Materials. Table S1 shows the solution method. The mesh checking results are shown in Figure 10. When the size of grids is less than $0.02 \mathrm{~mm}$, the temperature distribution in a radial direction is unchanged. In this paper, all meshes were map mesh and the average size of mesh was selected as $0.015 \mathrm{~mm}$. 


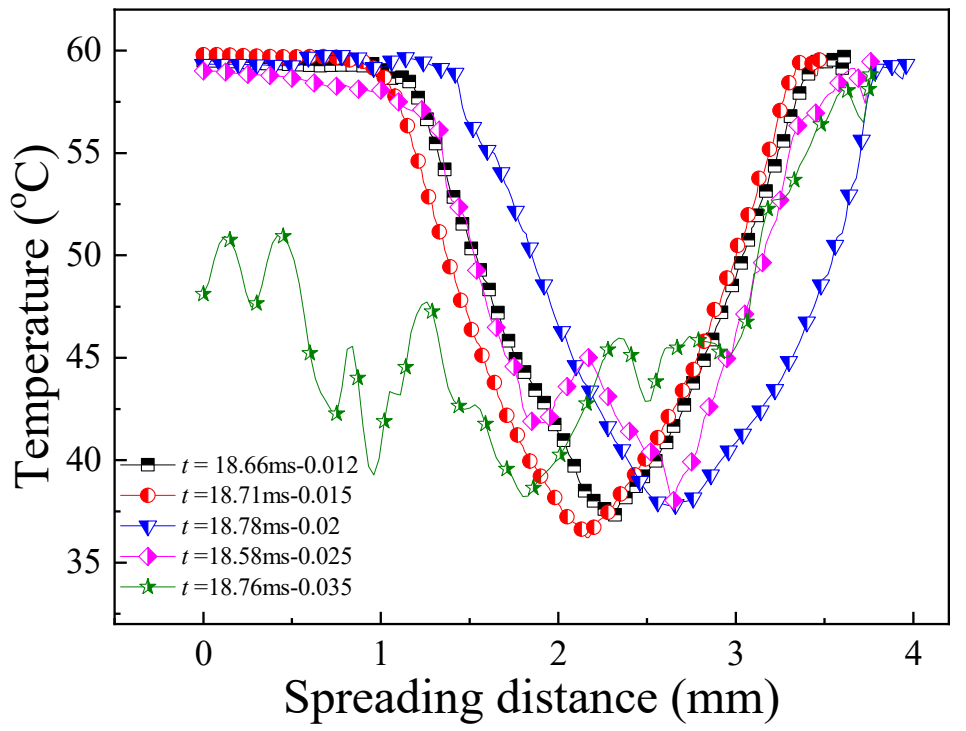

Figure 10. Mesh checking results: the comparison of radial temperature distribution on the air-liquid interface of simulation for $W e=16, T_{\mathrm{w}}=60^{\circ} \mathrm{C}$.

The right half of the droplet is simulated as shown in Figures 11 and 12, for $W e=16$, $T_{\mathrm{w}}=40{ }^{\circ} \mathrm{C}$. The inertial spreading and oscillating behaviors of the droplet on the hydrophilic copper surface are displayed in Figure 11. Once contacting the surface, the droplet will spread driven by inertia force to the maximum spreading diameter, and then oscillates under the action of surface tension and viscous force until reaching an equilibrium state eventually after a period of oscillation process. As shown in Figure 12, during the droplet impact and spreading process on the superhydrophilic surface with the same condition, the bottom of the droplet contacts with the surface first. Therefore, the temperature of the liquid will increase first and surface tension will decrease, and the droplet will spontaneously spread fast driven by inertial force under the effect of the surface tension decreasing, which results in a large part of the droplet leaves the center area. Additionally, when a large part of cold liquid moves to the boundary, which is called a congested region, the temperature distribution transformed into that a high temperature center area formed in the impact center surrounded by a ring-shaped the low-temperature zone. The phase distribution of the spreading process at $t=16 \mathrm{~ms}$ is shown in Figure $12 \mathrm{~b}$. There is the majority of initially cold droplet liquid to be collected and to form a thick liquid film. Thus, the effect of the heating process in that part is weaker than that in the center area. Therefore, the liquid film at the impact center is transported to the periphery along the spreading radius, and a ring-shaped the low-temperature region appears in the region where the liquid film gathers and congests, and an ultrathin liquid film appears naturally at the center of the impact which is the high-temperature center. A low-temperature ring zone and hot center will be formed by the droplet fluid leaving the impact center to flow nearby and congest, which is in agreement with the thermal tracing experimental results. 

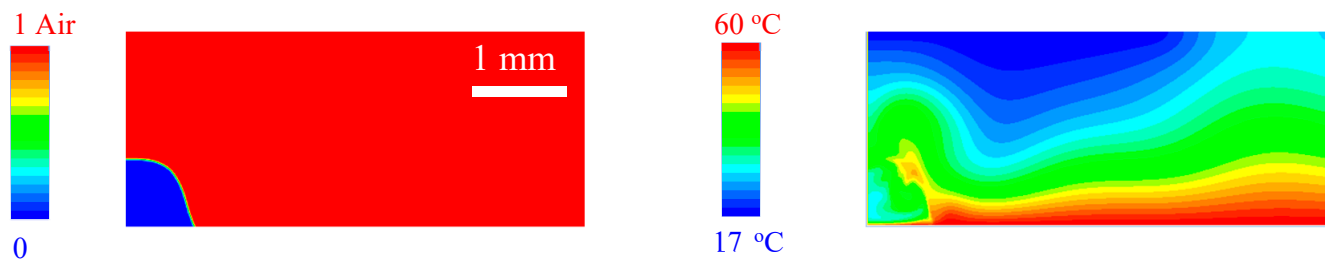

(a) $t=17 \mathrm{~ms}$
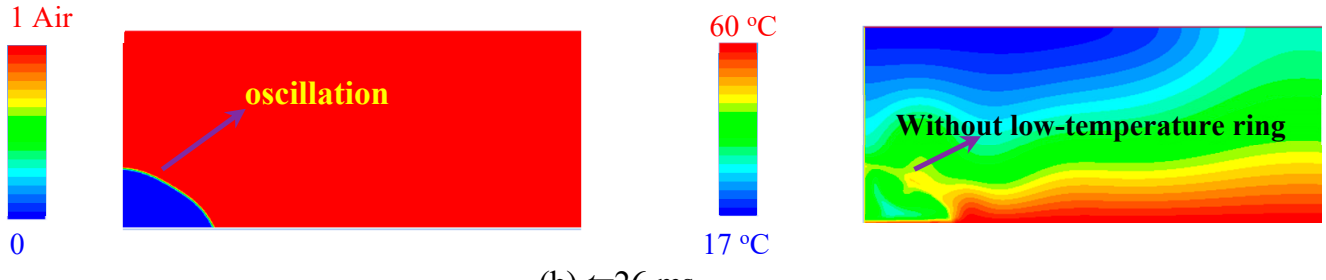

(b) $t=26 \mathrm{~ms}$

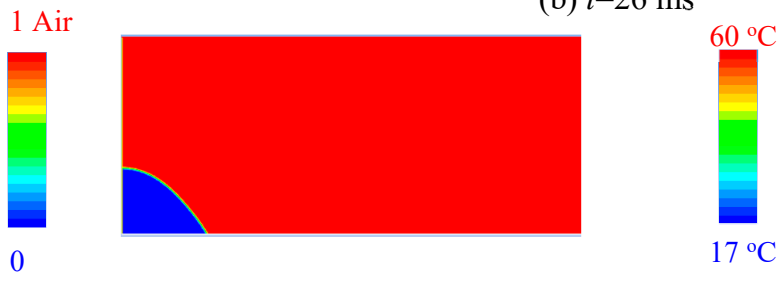

(c) $t=31 \mathrm{~ms}$

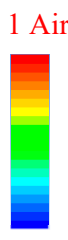

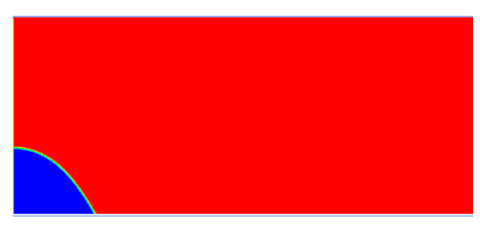

(d) $t=40 \mathrm{~ms}$

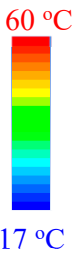

$17^{\circ} \mathrm{C}$

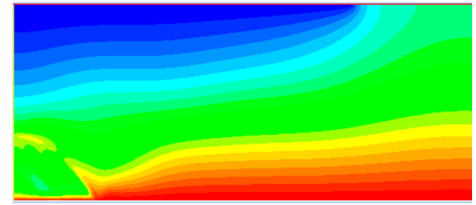

Figure 11. Phase and temperature distribution of the liquid film on hydrophilic surface for $W e=16$, $T_{\mathrm{W}}=60^{\circ} \mathrm{C}$ (a) $t=17 \mathrm{~ms},(\mathbf{b}) t=26 \mathrm{~ms},(\mathbf{c}) t=31 \mathrm{~ms},(\mathbf{d}) t=40 \mathrm{~ms}$. The bottom left corner is the droplet impact center.

As shown in Figure 13a, the liquid droplet on the hydrophilic surface moves downward and there is no vortex in the liquid droplet at $W e=16, T_{\mathrm{w}}=60^{\circ} \mathrm{C}$. However, the flow field in the liquid film is changed on a superhydrophilic surface at the same condition. When the liquid film is retracting, with the effect of the superhydrophilic surface, it is easy to create a counter-clockwise direction inward flow in the liquid film as shown in Figure 13b. Additionally, at the same time, due to the liquid film flow driven by decreasing surface tension, the liquid will move upward and encounter the retracting fluid and then form a clockwise vortex at the upper part. The formation of two vortexes inside the liquid film stabilizes the thick liquid film and slows the droplet retracting process, which leads to the stabilization of the low-temperature ring. Because of the vortex formation inside the liquid films, the ring-shaped temperature structure has appeared. The vortex formed at thicker liquid films, which hinders the spreading liquid film from the droplet. The larger vortex formation in the liquid film results in a weakening of lateral spreading droplet, making the droplet concentrated in the impact center. Thus, droplets would spend more energy overcoming the congesting effect of vortex velocity distribution for lateral spreading. The counter-clockwise-direction vortex in spreading liquid film is propitious to delaying the retraction of liquid film, which stabilizes the liquid film morphology in Figure 12b. That morphology of liquid film shows a ring-shaped the low-temperature zone in wall temperature distribution in the experiment results. With the decrease in the center liquid film thickness and accumulation of liquid film at the congested area, a high-temperature center forms and a low-temperature zone form nearby the boundary area. Subsequently, because the temperature difference has already formed, the hot center 
and the low-temperature zone would be stable for seconds. The low-temperature area vanishes until the congested liquid film is retraced by surface tension and the heat transfer process is finished.
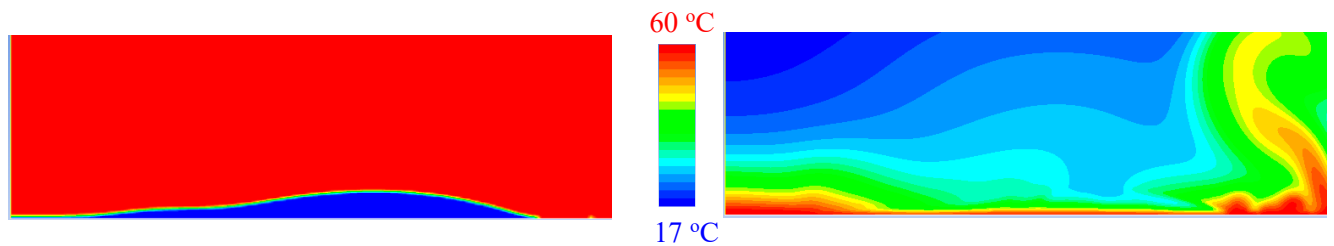

(a) $t=12 \mathrm{~ms}$
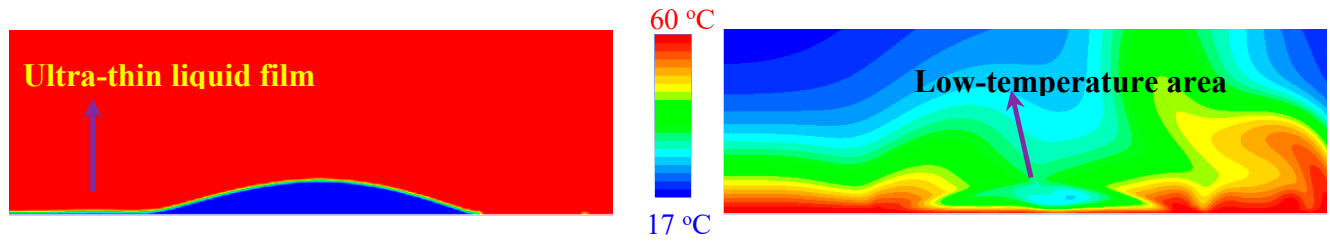

(b) $t=16 \mathrm{~ms}$
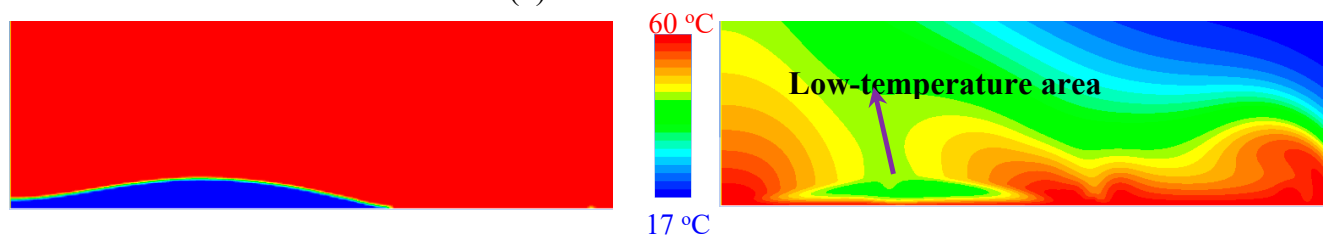

(c) $t=38 \mathrm{~ms}$
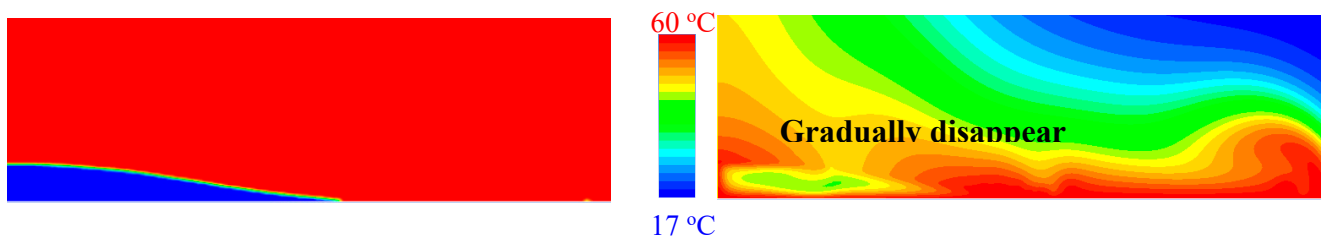

(d) $t=48 \mathrm{~ms}$

Figure 12. Phase and temperature distribution of the liquid film on superhydrophilic surface for $W e=16, T_{\mathrm{w}}=60^{\circ} \mathrm{C}$ (a) $t=12 \mathrm{~ms},(\mathbf{b}) t=16 \mathrm{~ms}$, (c) $t=38 \mathrm{~ms},(\mathbf{d}) t=48 \mathrm{~ms}$. The bottom left corner is the droplet impact center.

For droplet impact on a hydrophilic surface, the cold droplet remains in the impact center. The border area is heated quickly, and the temperature in the center is lowest and gradually increases from the center to the border. On a superhydrophilic surface, the spreading droplet behavior has changed, which results in the difference in temperature distribution after impact. The vortex velocity distribution in the liquid film of the superhydrophilic surface probably causes this special liquid film morphology and temperature distribution. It leads to the majority of the initial cold droplet to be collected and formed a thick liquid film region. The formation of the low-temperature ring structure is determined by the flow behavior of spreading liquid film on a heated superhydrophilic surface. 
(a)

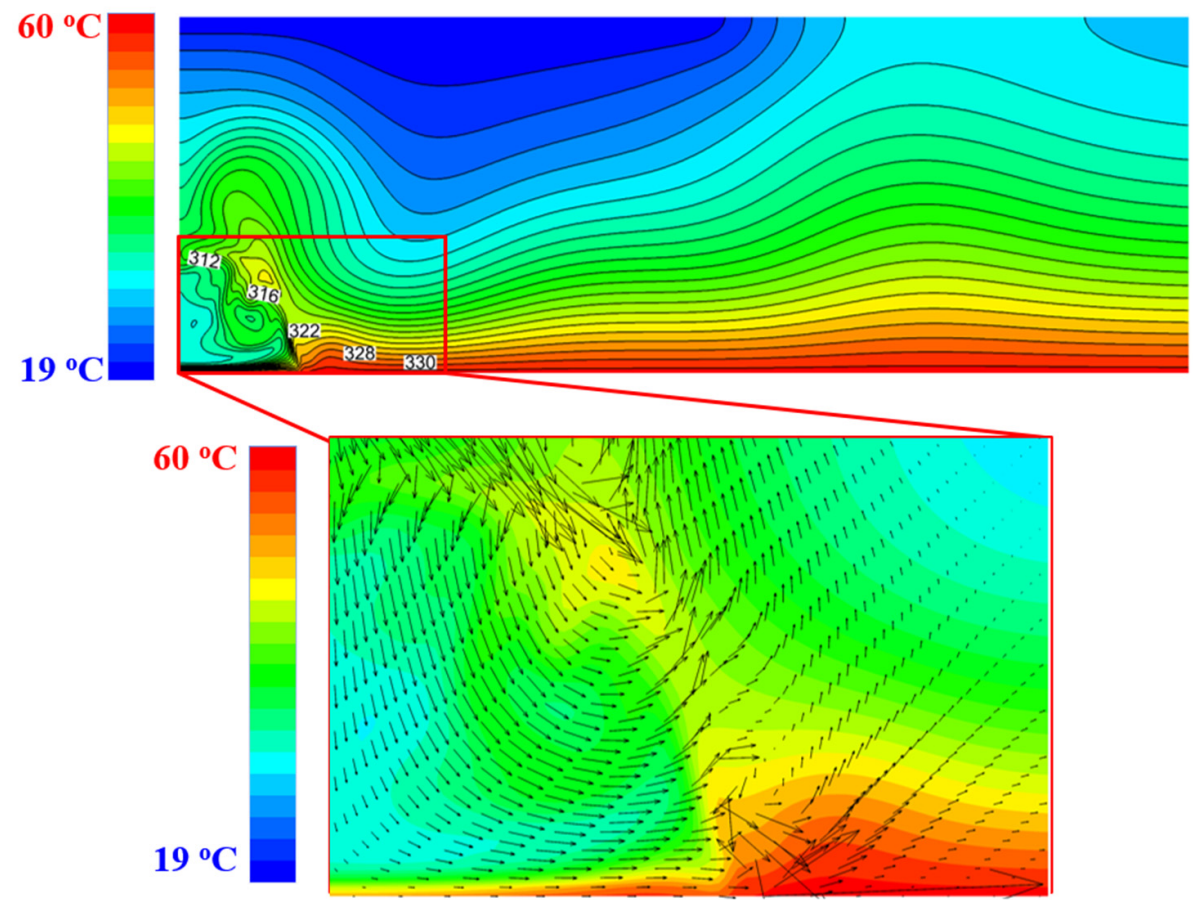

(b) 60

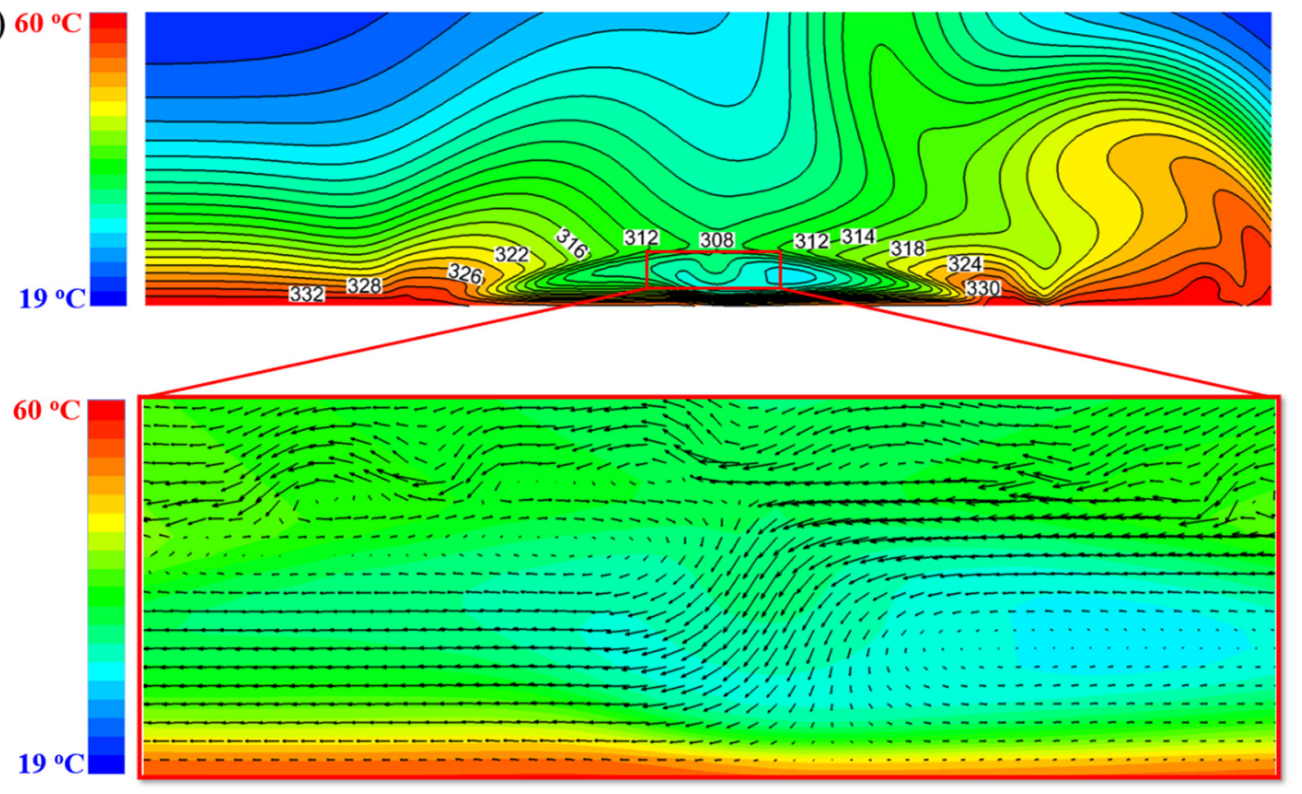

Figure 13. Isothermal curve and velocity vector distribution of the liquid film on (a) hydrophilic surface and (b) superhydrophilic surface at $t=17.75 \mathrm{~ms}$ for $W e=16, T_{\mathrm{w}}=60^{\circ} \mathrm{C}$.

\section{Conclusions}

The spreading characteristics and temperature evolution of droplets impacting the superhydrophilic surface were investigated by visualization experiments and numerical simulations. The temperature distribution after droplet impact on the heated superhydrophilic surface shows a ring-shaped the low-temperature zone, which is different from the gradient temperature distribution of droplet impact on the hydrophilic surface at the same We number and wall temperature. The formation of the low-temperature ring on the superhydrophilic surface transfers the low-temperature region from the thick to relatively thin region of liquid film, which facilitates the subsequent heat transfer process. The CFD numerical simulation is employed to clarify the mechanism of the low-temperature area formation. The ring-shaped temperature distribution is the result of the accumulation of 
cold thick liquid film which is caused by the vortex velocity distribution in liquid film at the superhydrophilic surface. The existence of vortex velocity distribution in liquid film stabilizes the thick liquid film at the congested zone. The heating surface effect in that region is weakened and leads to the formation of the low-temperature ring. While the spreading and retracting process continues, the low-temperature ring can exist in the whole process and move inward gradually until the liquid film temperature increases up to the wall temperature.

Supplementary Materials: The following are available online at https:/ /www.mdpi.com/article/10 .3390/coatings11091043/s1, Figure S1: Geometric model and boundary condition for CFD numerical simulation, Table S1: The numerical simulation parameters in Fluent 18.0.

Author Contributions: Conceptualization, H.M. and J.C.; methodology, H.M.; validation, F.Z., K.Q., and R.J.; data curation, H.M. and S.Z.; writing-original draft preparation, H.M. and J.C.; writing - review and editing, H.M. and J.C.; supervision, T.H. All authors have read and agreed to the published version of the manuscript.

Funding: The authors gratefully acknowledge the financial support for this work from the Program of National Natural Science Foundation of China (No.51836002).

Institutional Review Board Statement: Not applicable.

Informed Consent Statement: Not applicable.

Data Availability Statement: The data that support the findings of this study are available from the corresponding author upon reasonable request.

Conflicts of Interest: The authors declare no conflict of interest.

\section{References}

1. Zhang, Z.; Jiang, P.-X.; Christopher, D.M. Experimental investigation of spray cooling on micro-nano and hybrid-structured surfaces. Int. J. Heat Mass Transf. 2015, 80, 26-37. [CrossRef]

2. Zheng, Y.; Ma, X.; Li, Y. Experimental study of falling film evaporation heat transfer on superhydrophilic horizontal-tubes at low spray density. Appl. Therm. Eng. 2017, 111, 1548-1556. [CrossRef]

3. Worthington, A.M. On the forms assumed by drops of liquids falling vertically on a horizontal plate. Proc. R. Soc. Lond. 1876, 25, 261-272.

4. Yarin, A.L. Drop impact dynamics: Splashing, spreading, receding, bouncing ... . Annu. Rev. Fluid Mech. 2006, 38, 159-192. [CrossRef]

5. Rioboo, R.; Marengo, M.; Tropea, C. Time evolution of liquid drop impact onto solid, dry surfaces. Exp. Fluids 2002, 33, 112-124. [CrossRef]

6. Liang, G.; Mudawar, I. Review of drop impact on heated walls. Int. J. Heat Mass Transf. 2017, 106, 103-126. [CrossRef]

7. Wang, X.; Sun, D.; Wang, X.; Yan, W. Dynamics of droplets impacting hydrophilic surfaces decorated with a hydrophobic strip. Int. J. Heat Mass Transf. 2019, 135, 235-246. [CrossRef]

8. Tang, C.; Qin, M.; Weng, X. Dynamics of droplet impact on solid surface with different roughness. Int. J. Multiph. Flow 2017, 96, 56-69. [CrossRef]

9. Jin, Z.; Wang, Z.; Sui, D. The impact and freezing processes of a water droplet on different inclined cold surfaces. Int. J. Heat Mass Transf. 2016, 97, 211-223. [CrossRef]

10. Seol, H.K.A.; Gicheol, L.B.; Hyung, M.K.C.; Moo, H.K. Leidenfrost point and droplet dynamics on heated micropillar array surface. Int. J. Heat Mass Transf. 2019, 139, 1-9.

11. Pasandideh-Fard, M.; Aziz, S.D.; Chandra, S. Cooling effectiveness of a water drop impinging on a hot surface. Int. J. Heat. Fluid. Flow 2001, 22, 201-210. [CrossRef]

12. Fujimoto, H.; Oku, Y.; Ogihara, T. Hydrodynamics and boiling phenomena of water droplets impinging on hot solid. Int. J. Multiph. Flow 2010, 36, 620-642. [CrossRef]

13. Alizadeh, A.; Bahadur, V.; Zhong, S. Temperature dependent droplet impact dynamics on flat and textured surfaces. App. Phy. Lett. 2012, 11, 7699. [CrossRef]

14. Jin, Z.; Zhang, H.; Yang, Z. Experimental investigation of the impact and freezing processes of a water droplet on an ice surface. Int. J. Heat Mass Transf. 2017, 109, 716-724. [CrossRef]

15. Jin, Z.; Sui, D.; Yang, Z. The impact, freezing, and melting processes of a water droplet on an inclined cold surface. Int. J. Heat Mass Transf. 2015, 90, 439-453. [CrossRef]

16. Xiao, R.; Chu, K.H.; Wang, E.N. Multilayer liquid spreading on superhydrophilic nanostructured surfaces. App. Phy. Lett. 2009, 94, 988. [CrossRef] 
17. Lee, J.B.; Derome, D.; Carmeliet, J. Drop impact on natural porous stones. J. Colloid. Interf. Sci. 2016, 469, 147-156. [CrossRef] [PubMed]

18. Kim, S.J.; Moon, M.; Lee, K. Liquid spreading on superhydrophilic micropillar arrays. J. Fluid Mech. 2011, 680, 477-487. [CrossRef]

19. Kim, S.J.; Kim, J.; Moon, M.W. Experimental study of drop spreading on textured superhydrophilic surfaces. Phys. Fluids. 2013, 25, 092110. [CrossRef]

20. Ahn, H.S.; Park, G.; Kim, J. Wicking and spreading of water droplets on nanotubes. Langmuir 2012, 28, 2614-2619. [CrossRef] [PubMed]

21. Huang, Y.; Chen, Q.; Wang, R. Visualization study on capillary-spreading behavior of liquid droplet in vertically aligned carbon nanotube array. Int. J. Heat Mass Transf. 2018, 120, 1055-1064. [CrossRef]

22. Frank, X.; Perré, P.; Li, H.Z. Lattice Boltzmann investigation of droplet inertial spreading on various porous surfaces. Phys. Rev. E 2015, 91, 052405. [CrossRef]

23. Hengyi, K.; Lourenço, S.D.N.; Yan, W.M. Lattice Boltzmann simulation of droplet dynamics on granular surfaces with variable wettability. Phys. Rev. E 2018, 98, 012902.

24. Frank, X.; Perré, P. Droplet spreading on a porous surface: A lattice Boltzmann study. Phys. Fluids 2012, 24, 338. [CrossRef]

25. Choi, M.; Son, G.; Shim, W. A level-set method for droplet impact and penetration into a porous medium. Comput. Fluids 2017, 145, 153-166. [CrossRef]

26. Masumeh, F.S.; Mahmood, F.; Farshad, E. Contact angle hysteresis and motion behaviors of a water nano-droplet on suspended graphene under temperature gradient. Phys. Fluids 2018, 30, 052101.

27. Song, F.H.; Li, B.Q.; Liu, C. Molecular dynamics simulation of nanosized water droplet spreading in an electric field. Langmuir 2013, 29, 4266-4274. [CrossRef]

28. Chen, X.; Chen, J.; Ouyang, X. Water droplet spreading and wicking on nanostructured surfaces. Langmuir 2017, 33 , 6701. [CrossRef]

29. Wemp, C.K.; Carey, V.P. Water wicking and droplet spreading on randomly structured thin nanoporous layers. Langmuir 2017, 33, 14513. [CrossRef]

30. Wang, Z.; Espín, L.; Bates, F.S. Water droplet spreading and imbibition on superhydrophilic poly(butylene terephthalate) melt-blown fiber mats. Chem. Eng. Sci. 2016, 146, 104-114. [CrossRef]

31. Espin, L.; Kumar, S. Droplet spreading and absorption on rough, permeable substrates. J. Fluid Mech. 2015, 784, 465-486. [CrossRef]

32. Girard, F.; Antoni, M.; Sefiane, K. Infrared thermography investigation of an evaporating sessile water droplet on heated substrates. Langmuir 2010, 26, 4576. [CrossRef]

33. Gao, X.; Kong, L.; Li, R.; Han, J. Heat transfer of single drop impact on a film flow cooling a hot surface. Int. J. Heat Mass Transf. 2017, 108, 1068-1077. [CrossRef]

34. Li, Y.; Zheng, Y.; Lan, Z. The evolution of droplet impacting on thin liquid film at superhydrophilic surface. App. Phys. Lett. 2017, 111, 231601. [CrossRef]

35. Li, Y.; Zheng, Y.; Lan, Z. The exact regulation of temperature evolutions for droplet impact on ultrathin cold films at superhydrophilic surface. Chem. Eng. Sci. 2017, 193, 205-216. [CrossRef]

36. Zheng, Y.; Lan, Z.; Cao, K. A new insight of temperature distribution on superhydrophilic surface horizontal tubes falling film at low spray density. Int. Comm. Heat Mass Transf. 2018, 91, 17-22. [CrossRef]

37. Youngsuk, N.Y.; Sungtaek, J. A comparative study of the morphology and wetting characteristics of micro/nanostructured Cu surfaces for phase change heat transfer applications. J. Adhes. Sci. Technol. 2013, 27, 2163-2176. 